\title{
Revista Colombiana de

\section{Complicaciones de la diabetes y su asociación con el estrés oxidativo: un viaje hacia el daño endotelial}

\author{
Marcelo A. Storino ${ }^{\mathrm{a}, *}$, Miguel A. Contreras ${ }^{\mathrm{b}}$, Jairo Rojano ${ }^{\mathrm{c}}$, \\ Richard Serrano ${ }^{d}$ y Ana Nouel ${ }^{d}$ \\ a Medicina Interna, Asesoría Médica de AstraZeneca Venezuela, Clínica Fénix Salud Caracas, Venezuela \\ ${ }^{\mathrm{b}}$ Medicina Interna. Clínica Chico Sanabria, Porlamar - Margarita, Venezuela \\ c Medicina Interna - Reumatología. Hospital Dr. Miguel Pérez Carreño Caracas, Venezuela \\ d Medicina Interna. Hospital Dr. Miguel Pérez Carreño Caracas, Venezuela
}

Recibido el 3 de febrero de 2014; aceptado el 11 de septiembre de 2014

Disponible en Internet el 11 de diciembre de 2014

\author{
PALABRAS CLAVE \\ Radicales libres; \\ Diabetes mellitus; \\ Endotelio; \\ Glucosa; \\ Vasos; \\ Respuesta \\ inflamatoria
}

\begin{abstract}
Resumen La diabetes es un trastorno metabólico que se ha incrementado en Latinoamérica y Venezuela en la última década. Ejerce una influencia considerable en la morbimortalidad de las enfermedades cardiovasculares debido al desequilibrio hidrocarbonado y a los pacientes fuera de los objetivos de la Asociación americana de diabetes 2012, con factores de riesgo como hipertensión y dislipidemia.

Es, igualmente, un estado de estrés oxidativo en el que hay un desequilibrio entre la formación excesiva y la eliminación insuficiente de moléculas altamente reactivas, como las especies reactivas de oxígeno y las de nitrógeno. El estrés oxidativo desempeña un papel importante en el desarrollo de complicaciones de la diabetes debido a la actividad oxidativa excesiva.

La disfunción endotelial se define como alteraciones que afectan la síntesis, liberación, difusión o degradación de los factores sintetizados por el endotelio. El mecanismo intrínseco que impulsa a las células endoteliales para cambiar su fenotipo fisiológico es un estado de activación, conocido erróneamente como disfunción en la capacidad de expresar los sistemas enzimáticos de producción de oxígeno.

Los fármacos que modulan la vía de la incretina (GLP-1 y los inhibidores de la DPP-4) se caracterizan por la baja incidencia de su efecto hipoglucemiante, fenómeno que aumenta su seguridad. Estudios recientes con GLP-1 demuestran su capacidad para revertir el estrés oxidativo producido por la hiperglucemia, la hipoglucemia y la transición de esta última y la primera.

(c) 2014 Publicado por Elsevier España, S.L.U. en nombre de Sociedad Colombiana de Cardiología y Cirugía Cardiovascular.
\end{abstract}

\footnotetext{
* Autor para correspondencia.

Correo electrónico: Marcelo.Storino@astrazeneca.com (M.A. Storino).
} 


\section{KEYWORDS}

Free radical;

Diabetes mellitus;

Endothelium;

Glucose;

Vessels;

Inflammatory

response
Complications of diabetes and its association with oxidative stress: a journey to the endothelial damage

Abstract Diabetes is a metabolic disorder that has increased in Latin America and Venezuela over the last decade. It exerts an important influence on cardiovascular diseases morbimortality because of the hydrocarbonate imbalance and on patients outside ADA's 2012 goals, with risk factors such as hypertension and dyslipidemia.

Diabetes is a state of oxidative stress where there is an imbalance between excessive formation and insufficient removal of highly reactive molecules such as reactive oxygen species (ROS) and reactive nitrogen species (RNS). Oxidative stress plays an important role in developing diabetes complications because excessive oxidative activity.

Endothelial dysfunction is defined as alterations affecting synthesis, liberation, diffusion or degradation of the factors synthesized by the endothelium. The intrinsic mechanism that drives endothelial cells to switch their physiologic phenotype into an activation state mistakenly known as dysfunction is the ability to express $\mathrm{O}_{2}$ production enzymatic systems.

Drugs that modulate incretin pathway (GLP-1 and DPP4 inhibitors) are characterized by their low incidence of hypoglycemic effect, which increases their safety. Recent studies with GLP-1 have shown its ability to reverse oxidative stress produced by hyperglycemia, hypoglycemia and the transition from hypo to hyperglycemia.

(c) 2014 Published by Elsevier España, S.L.U. on behalf of Sociedad Colombiana de Cardiología y Cirugía Cardiovascular.

\section{Introducción}

La diabetes mellitus es un desorden metabólico con gran incidencia mundial, del que se ha registrado un incremento en la última década en Latinoamérica y Venezuela, como lo demuestran Schargrodsky et al. ${ }^{1}$ en su trabajo sobre «Factores de Riesgo Cardiovascular en América Latina", (estudio CARMELA), donde alcanza una prevalencia del 7\% en general, si bien al analizar solo la información recolectada en Barquisimeto, del mismo grupo de investigación, se obtiene una prevalencia del $6 \%^{1}$ (tabla 1 ).

Sin embargo, el problema no solo está en la prevalencia de la enfermedad sino en el alto número de pacientes que viven con la patología y que están fuera de las metas de hemoglobina glucosilada (HbA1c), como se expresa en el trabajo de Moreira et al. ${ }^{6}$ donde el promedio de la HbA1c en 4.096 pacientes con diabetes fue de $9,11 \%$ en Venezuela ${ }^{6}$, hecho que aporta una perspectiva de cómo puede influir la diabetes mellitus en el incremento de la morbimortalidad por enfermedades cardiovasculares (tabla 2), ya que no son solo factores de riesgo la hipertensión o dislipidemia, sino también el desorden hidrocarbonado y los pacientes muy fuera de las metas establecidas por la Asociación americana de diabetes del $2012^{7}$.

De otra parte, la diabetes no es solo un trastorno hidrocarbonado, pues también se conoce como un estado de estrés oxidativo ${ }^{3}$, en el que se da un disbalance entre la formación excesiva y la remoción insuficiente de moléculas altamente reactivas como las especies reactivas de oxígeno (ROS) y las especies reactivas de nitrógeno (RNS). En este grupo se incluyen, además, dos especies muy conocidas por su relación con el endotelio: el ion superóxido $\left(\mathrm{O}_{2}^{-}\right)$y su contraparte, el óxido nítrico (NO-), las cuales se abordarán en esta revisión ${ }^{3}$.
Tabla 1 Factores de riesgo cardiovascular en Venezuela (Estudio CARMELA)

Prevalencia (\%) de los factores de riesgo cardiovascular (media e intervalo)

\begin{tabular}{ll}
\hline Hipertensión $^{2}$ & 25 \\
Dislipidemia $^{3}$ & 70 \\
Diabetes o AGA $^{4}$ & 6 \\
Síndrome metabólico $^{5}$ & 26 \\
Obesidad abdominal $(\mathrm{h} / \mathrm{m})_{\text {Hipertrigliceridemia }(\mathrm{h}(\mathrm{m})}^{57 / 84}$ \\
Reducción c-HDL $(\mathrm{h} / \mathrm{m})$ & $90 / 79$ \\
Hipertensión $(\mathrm{h} / \mathrm{m})$ & $93 / 96$ \\
Diabetes o AGA $(\mathrm{h} / \mathrm{m})$ & $82 / 64$ \\
\hline
\end{tabular}

Fuente: Barquisimeto 1.848 sujetos; edad media $45 \pm 11$ años, $61 \%$ varones, $39 \%$ mujeres. Tomada de Schargrodsky et al. ${ }^{1}$.

\section{Efecto de la oxidación en el endotelio}

Las células del endotelio vascular cumplen un papel decisivo para mantener la homeostasis cardiovascular. Además de proveer una barrera física en la pared vascular y el lumen $^{8}$, el endotelio se considera en la actualidad un órgano perteneciente al sistema neuroendocrino difuso, cuyas células ejercen funciones vasodilatadoras y vasoconstrictoras, anticoagulantes y procoagulantes, antiinflamatorias y proinflamatorias, proliferativas y antiproliferativas, angiogénicas y antiangiogénicas, inmunes y autoinmunes, y autocrinas, paracrinas y endocrinas; y sintetizan disímiles moléculas con funciones vasoactivas y hormonales, neuropéptidos, neurotransmisores, citoquinas, factores de crecimiento, factores quimioatrayentes, moléculas de adhesión celular 
Tabla 2 Mortalidad por enfermedades del corazón en Venezuela. Año 2010

\begin{tabular}{llr}
\hline Causa de muerte & Mortalidad diagnosticada & Porcentaje \\
\hline Enfermedades del corazón & 29.078 & 20,99 \\
Infarto agudo de miocardio & 18.752 & 13,54 \\
Enfermedad cardiaca hipertensiva & 3.013 & 2,18 \\
Enfermedad isquémica crónica del corazón & 2.947 & 2,18 \\
\hline
\end{tabular}

Fuente: Gobierno Bolivariano de Venezuela ${ }^{4}$.

y receptores de membrana, lo que contribuye a convertirlo en el órgano más versátil del organismo humano ${ }^{9,10}$.

La disfunción endotelial cumple un papel preponderante en la génesis de las complicaciones vasculares de la diabetes y precede a las evidencias anatómicas más tempranas de aterosclerosis $^{11}$.

El concepto de disfunción endotelial implica un daño funcional, el cual puede evolucionar hacia estructural ${ }^{12}$, si bien en otras literaturas se considera como un trastorno en el cual este pierde sus propiedades fisiológicas: la tendencia a favorecer la vasodilatación, la fibrinólisis y la antiagregación ${ }^{8}$. Sin embargo, la disfunción endotelial debe diferenciarse de la activación endotelial, la cual es una respuesta fisiológica cuyo objetivo es compensar y/o anular los efectos nocivos de un estímulo potencialmente lesivo a los tejidos ${ }^{12}$.

De forma general, se puede definir la disfunción endotelial (DE) como la serie de alteraciones que afectan la síntesis, la liberación, la difusión o la degradación de los factores que se sintetizan por el endotelio ${ }^{9}$. La DE no es homogénea en sus características ni en su distribución; estos aspectos varían a expensas de la enfermedad que esté presente, así como del lecho vascular afectado ${ }^{9,10,13}$.

La etapa inicial de la disfunción endotelial involucra el reclutamiento de células inflamatorias y su migración a través del endotelio. Este proceso está regulado por moléculas de adhesión que se expresan en la superficie de leucocitos y células endoteliales, y que tendrían un papel trascendental en la predicción de los eventos cardiovasculares ${ }^{11}$. El mecanismo intrínseco que le permite a la célula endotelial «switchear» su fenotipo fisiológico en uno de activación mal conocido como disfunción, es la capacidad de expresión de sistemas enzimáticos productores de oxígeno, incluyendo a la eNOS desacoplada o «uncoupled» NOS-uNOS- y la puesta en marcha de factores de transcripción inflamatorios como el NFkB ${ }^{12}$.

Entre las moléculas de adhesión que intervienen están las selectinas (E-selectina [E-S] y P-selectina), las moléculas de adhesión intracelular (ICAM-1) y las moléculas de adhesión vásculo-celular (VCAM-1). La E-S es una glicoproteína que, a diferencia de ICAM-1 y VCAM-1, se expresa exclusivamente en las células endoteliales activadas, en respuesta a citoquinas inflamatorias como la interleuquina-1 beta (IL-1 $\beta$ ), $y / o$ el factor de necrosis tumoral alfa (TNF- $\alpha)$. Se ha sugerido que niveles aumentados de formas solubles de E-S (sE-S) podrían ser marcadores específicos y precoces de endotelio vascular dañado ${ }^{11}$.

La célula endotelial expresa en su membrana receptores específicos para colesterol-LDL oxidado o LOX-R, angiotensina ॥ o AT1-R y productos de glucosilación avanzada o RAGEs. La unión de los diferentes ligandos a sus receptores, activa a los sustratos de membrana asociados en su mayoría de proteínas $G$ y proteínas $G$ pequeñas -smg-. Así, las interacciones ligando-receptor, inician cascadas enzimáticas cuya vía final común es la producción de radicales superóxido, especialmente oxígeno. El sistema de la NADPH oxidasa y la eNOS desacoplada -uNOS- son los sistemas productores de radicales superóxido en la célula endotelial más importantes; estas moléculas son el indicador intracelular de una lesión extracelular. El incremento citoplasmático de oxígeno hace que estos reaccionen con el óxido nítrico y lo transformen en peroxinitrito, induzcan proteólisis por ubiquitinación del IkB y rompan el «secuestro» de la familia de factores de transcripción incluidos en el NFkB, cada uno de los cuales iniciará la transcripción, síntesis y expresión de diversas moléculas inflamatorias. De esta manera, la célula endotelial activada en su genotipo inflamatorio producirá decenas de moléculas, cuyo efecto biológico reflejará las diferentes facetas de lo que se ha denominado «fenotipo de disfunción endotelial» ${ }^{12}$.

La interacción de los productos finales de la glucosilación avanzada con sus receptores celulares promueve la producción intracelular de RL y contribuye a disminuir los niveles intracelulares de antioxidantes. Asimismo, el glioxal, especie derivada de la oxidación de la glucosa, puede generar citotoxicidad mediada por incremento de la generación de EROs y disminución del GSH intracelular. Por otro lado, la glucación de las proteínas antioxidantes puede disminuir la actividad de estas mientras que la hemoglobina glucada puede constituir una fuente donadora de radical $\mathrm{O}_{2}-$ a la pared vascular en los diabéticos ${ }^{14-16}$.

En el estado diabético, la acumulación excesiva de $\mathrm{O}_{2}{ }^{\circ}$ y el incremento consiguiente de la actividad de la proteína quinasa $\mathrm{C}$ y del flujo de las hexosaminas provocan disfunción celular progresiva ${ }^{14}$.

Velarde et al., realizaron un estudio en el que determinaron los niveles de sE-S y ET-1 en diabéticos tipo 2 y relacionaron la posible asociación de dichas moléculas con otros factores de riesgo cardiovascular como índice de masa corporal elevado, hipertensión arterial, hipercolesterolemia y grado de control glucémico. Determinaron que estos pacientes presentaron niveles elevados de SE-S y ET-1 y moléculas marcadoras de activación endotelial. Los diabéticos con índice de masa corporal alto presentaron un incremento significativo de sE-S y ET-1, que potenciaba la disfunción endotelial en estos. El grado de control glucémico se asoció con niveles elevados de ET-1, lo que confirmaría que la hiperglucemia crónica afecta la vasodilatación dependiente del endotelio. Estos resultados sugieren que la detección temprana de marcadores bioquímicos de disfunción endotelial contribuiría a la prevención de enfermedad cardiovascular en pacientes de alto riesgo ${ }^{11}$. 
Por otra parte, existen varios mecanismos implicados en el incremento del estrés oxidativo en la diabetes mellitus, entre los cuales se encuentran: la autooxidación de la glucosa, la glucación de proteínas, la activación de la vía de los polioles y la disminución de las defensas antioxidantes. La glucosa, al igual que otros alfahidroxialdehídos, es capaz de autooxidarse a enedioles (enolizarse) en solución acuosa y en presencia de metales de transición, como el $\mathrm{Fe}_{3}^{+}$, reacción en la cual se producen citoaldehídos intermediarios oxidados y $\mathrm{RL}$, con alto poder oxidante como el $\mathrm{O}_{2}{ }^{\circ}{ }_{-14,17,18}$.

En términos generales, la modificación oxidativa de las proteínas incrementa su degradabilidad y susceptibilidad a la proteólisis, probablemente por el aumento de su hidrofobicidad, lo cual implica, específicamente, una más rápida ubiquitinización y degradación por la vía lisosomal ${ }^{14}$. La retina es el tejido neurosensorial del ojo y es extremadamente rica en membranas con lípidos poliinsaturados, característica que la hace especialmente sensible a la acción deletérea de los RL derivados del oxígeno y el nitrógeno ${ }^{14}$. Un blanco frecuente del ataque de los RL en el riñón son los lípidos de las membranas de las células renales, lo cual provoca su peroxidación y altera la integridad y la función de estas membranas ${ }^{14,19}$.

El contenido de carbonilos proteicos es el marcador más utilizado para medir la modificación oxidativa de las proteínas y se ha sugerido que es un marcador confiable de estrés oxidativo.Asimismo, el contenido de carbonilos proteicos se correlaciona positivamente con las complicaciones de la diabetes ${ }^{14,20,21}$.

Kowluru estudió ratas en las que se indujo diabetes mediante la inyección de aloxano, y demostró que en la retina de estas había un aumento de la concentración de sustancias reactivas al ácido tiobarbitúrico y de óxido nítrico (ON), y un incremento de la actividad de la proteína quinasa C, además de una disminución de la concentración de GSH. Por su parte, Du et al. demostraron que la hiperglucemia incrementa la producción de $\mathrm{ON}$ en las células retinianas, lo que se debe al aumento de la actividad de la ON sintetasa. Este aumento de ON se acompaña de la generación de peroxinitrito, el cual está implicado en la aparición de apoptosis en las células nerviosas de la retina ${ }^{14,22}$.

En los nervios, esta confluencia de alteraciones metabólicas y vasculares produce trastornos en la función neuronal y favorece la pérdida del soporte neurotrófico y, a largo plazo, la aparición de la apoptosis de las neuronas, de las células de Schwann y de las células gliales ${ }^{14,23,24}$.

Muchos han sido los intentos por encontrar un marcador universal lo suficientemente preciso y específico de DE. Así, trató de considerarse como tal a la elevación de las células endoteliales maduras circulantes y de las micropartículas endoteliales, pequeñas vesículas formadas por la membrana celular, que son liberadas por las células endoteliales activadas o dañadas, y a la disminución de las células endoteliales progenitoras circulantes, pero hasta el momento esto no puede considerarse ciencia constituida. En la actualidad, para identificar la presencia de DE, se utilizan la medición de sustancias reguladoras de biofunciones sintetizadas por el endotelio y de otras reconocidas como marcadores de DE, y pruebas indirectas, cuyos métodos se fundamentan en la respuesta vasoactiva a drogas (vasodilatación con acetilcolina) o a estímulos mecánicos, y para la realización de las cuales es preciso auxiliarse, en algunos casos, de técnicas invasivas como la coronariografía. Por otro lado, entre estas pruebas hay algunas que utilizan métodos no invasivos, como la ultrasonografía de alta resolución (eco doppler vascular) y la tomografía por emisión de positrones ${ }^{9,13,25}$.

\section{Implicaciones sistémicas del estrés oxidativo en la hiperglucemia}

Una de las formas de poder entender este punto, es asociar las diferentes complicaciones crónicas de la diabetes, sobre todo cuando existe un estado de hiperglucemia, y relacionarlas con los diferentes estados de estrés oxidativo que se están dando a lugar. El estrés oxidativo ejerce un rol determinante en el desarrollo de complicaciones de la diabetes mellitus ${ }^{4}$. La actividad excesiva de oxidante está mecánicamente relacionada con diversas etiologías de la resistencia a la insulina y el desarrollo de diabetes mellitus tipo $2^{5}$. Hay muchas fuentes de estrés oxidativo en diabetes mellitus, incluida la enzimática, las vías no enzimáticas y la mitocondrial. El aumento de estrés oxidativo en la diabetes mellitus se produce debido a diversos factores ${ }^{26}$. El factor más dominante múltiple es la glucosa autooxidación que resulta en el desarrollo de los radicales libres ${ }^{27}$.

La diabetes mellitus también se caracteriza por complicaciones de microangiopatía, tales como retinopatía, nefropatía y neuropatía ${ }^{28}$. Varios estudios demuestran que la neutralización de moléculas reactivas ha sido capaz de inhibir significativamente el desarrollo de disfunción endotelial, miocardiopatía, retinopatía, nefropatía y neuropatía en pacientes con diabetes mellitus ${ }^{29}$ (fig. 1).

En este punto se introduce el papel de las plaquetas en el esquema oxidativo, y el término de alta reactividad plaquetaria (HPR) y su relación con la diabetes mellitus ${ }^{30}$. Al respecto, sobresalen los trabajos de Rosiak et al. ${ }^{30}$ quienes estudiaron pacientes diabéticos expuestos a antiagregación con aspirina (AAS) a dosis simple (75 mg) y duplicada (150 $\mathrm{mg}$ ) más medición de HPR, entre otros parámetros como el trombaxano $A_{2}\left(T X B_{2}\right)$; es un subanálisis del estudio $\mathrm{AVOCADO}^{30}$. En resumen, la duplicación de la dosis de AAS en pacientes que presentan HPR se asoció con mejoría significativa en la reactividad de las plaquetas ${ }^{30}$.

\section{La hipoglucemia y su relación con el estrés oxidativo}

La hipoglucemia, al igual que la hiperglucemia, se ha asociado, de forma cada vez más frecuente, con enfermedad cardiovascular independientemente de que se presente en forma aguda o crónica. Dicha hipoglucemia forma parte de las complicaciones del tratamiento de la diabetes, tanto con el uso de insulina, como de hipoglucemiantes orales, y su incidencia aumenta de forma exponencial después de ocho a diez años de evolución de la enfermedad ${ }^{31}$.

Los estudios más recientes que evaluaron el efecto sobre la morbimortalidad cardiovascular del tratamiento intensivo de la diabetes, han detectado efectos muy modestos, o aún más, efectos negativos de esta estrategia, hecho que podría reflejar el efecto negativo de la hipoglucemia sobre el sistema cardiovascular ${ }^{31}$ (fig. 2). 


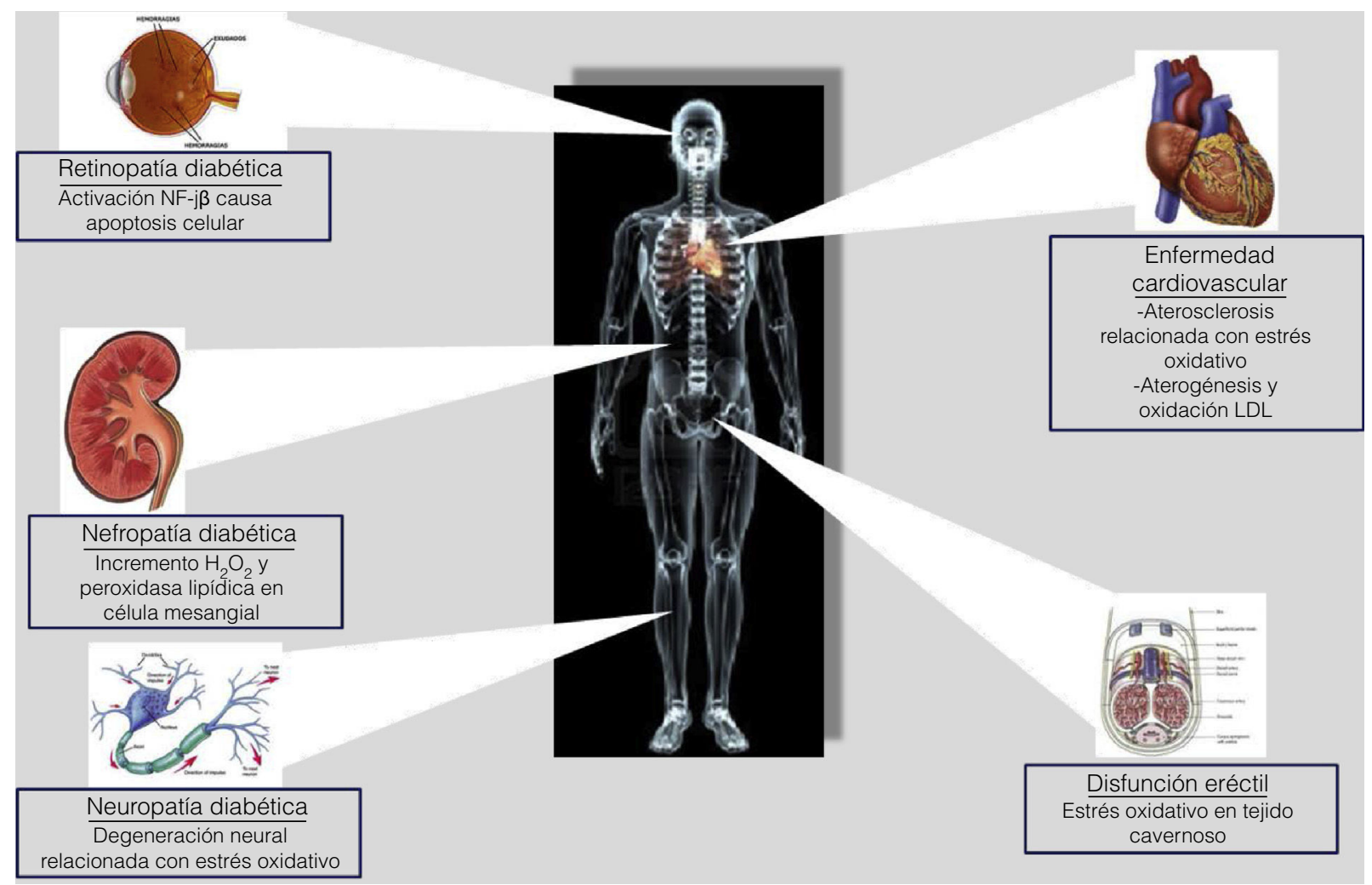

Figura 1 Complicaciones de la diabetes y su asociación con el estrés oxidativo.

Llama la atención que la hipoglucemia ejerce efectos muy similares a los que produce la hiperglucemia sobre el endotelio $^{32}$ (fig. 3):

a. Aumenta la producción de radicales libres (estrés oxidativo) y biomarcadores inflamatorios como la proteína $C$ reactiva y las interleucinas 6 y $8^{33}$.

b. Favorece la agregación plaquetaria, la producción del factor vill, el factor de Von Willebrand y los procesos implicados en la aterotrombosis ${ }^{31}$.

c. Potencia la vasoconstricción y la producción de endotelina ${ }^{34}$

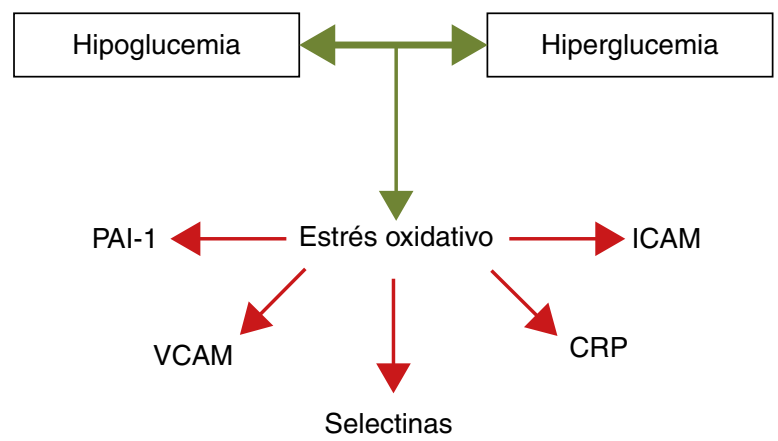

Fuente: diseñada por Contreras

Figura 2 Efecto generador de estrés oxidativo e inflamación de la hipo- e hiperglucemia. d. De forma aguda, potencia la respuesta simpáticoadrenérgica con aumento de la incidencia de arritmias y muerte cardiaca súbita ${ }^{31}$.

Ahora bien, con base en lo anterior ¿cuáles son los mecanismos por medio de los cuales se producen dichos efectos?

En primer lugar, debe tenerse en cuenta que la hipoglucemia favorece la liberación de mediadores adrenérgicos, cortisol y ácidos grasos libres que, de forma paradójica, potencian la insulinorresistencia y la inflamación concomitante ${ }^{35-37}$.

Por otra parte, la hiper e hipoglucemia generan estrés oxidativo con liberación de radicales libres y disfunción endotelial. Aún más, la situación que produce mayor

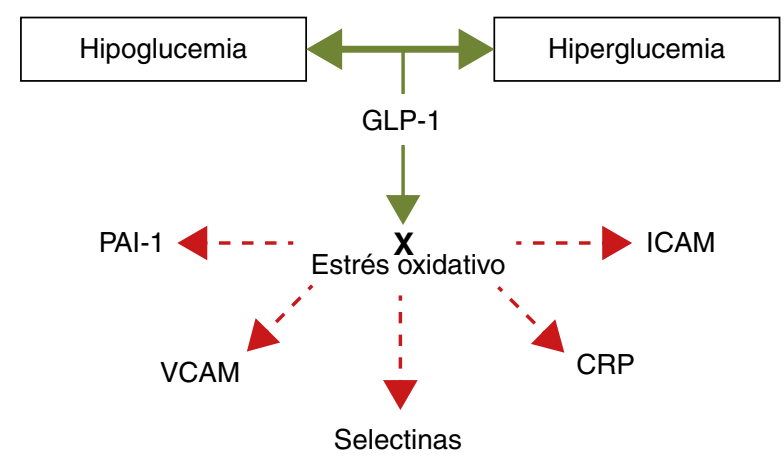

Fuente: diseñada por Contreras

Figura 3 Efecto protector vascular del GLP-1 sobre el endotelio. 
generación de radicales libres es el paso brusco de hipo a hiperglucemia, lo cual revela un mecanismo etiopatológico común para las diferentes situaciones características de la disglucemia.

Los medicamentos que modulan la vía de las incretinas (GLP-1 e inhibidores DPP4) se caracterizan por su baja incidencia de hipoglucemia, lo cual incrementa la seguridad de su manejo. Pero, como efecto añadido, los estudios más recientes con GLP-1 han demostrado que es capaz de revertir el estrés oxidativo producido por la hiper y la hipoglucemia y por el paso de hipo a hiperglucemia ${ }^{38-40}$. Este mecanismo podría ayudar a explicar el efecto cardioprotector demostrado en los más recientes metaanálisis de esta línea de medicamentos.

Como puede observarse, la hipoglucemia constituye la «otra cara de la moneda» del efecto aterotrombótico de la glucosa y, de no tomarse en cuenta este punto, no se estará cubriendo el espectro completo del daño vascular.

\section{Conflicto de intereses}

Marcelo Storino es Asesor Médico de AstraZeneca de Venezuela.

\section{Bibliografía}

1. Schargrodsky H, Hernández-Hernández R, Champagne BM, Silva H, Vinueza R, Silva Ayçaguer LC, et al., CARMELA Study Investigators. CARMELA: assessment of cardiovascular risk in seven Latin American cities. Am J Med. 2008;121(1):58-65.

2. Gobierno Bolivariano de Venezuela. Anuario de Mortalidad 2010. Caracas. Venezuela: Ministerio del Poder Popular para la Salud; 2012.

3. Rahimi R, Nikfar S, Larijani B, Abdollahi M. A review on the role of antioxidants in the management of diabetes and its complications. Biomed Pharmacother. 2005;59:365-73.

4. Giacco F, Brownlee M. Oxidative stress and diabetic complications. Circ Res. 2010;107:1058-70.

5. Henriksen EJ, Diamond-Stanic MK, Marchionne EM. Oxidative stress and the etiology of insulin resistance and type 2 diabetes. Free Radic Biol Med. 2010 (Epub ahead of print).

6. Moreira ED Jr, Neves RC, Nunes ZO, de Almeida MC, Mendes AB, Fittipaldi JA, et al., Venezuelan Diabetes Investigators' Group. Glycemic control and its correlates in patients with diabetes in Venezuela: results from a nationwide survey. Diabetes Res Clin Pract. 2010;87(3):407-14.

7. Inzucchi SE, Bergenstal RM, Buse JB, Diamant M, Ferrannini E, Nauck M, et al.; American Diabetes Association (ADA); European Association for the Study of Diabetes (EASD). Management of hyperglycemia in type 2 diabetes: a patient-centered approach: position statement of the American Diabetes Association (ADA) and the European Association for the Study of Diabetes (EASD). Diabetes Care. 2012;35:1364-79.

8. Avogaro A, Albiero M, Menegazzo L, Kreutzenberg S, Fadini GP. Disfuncion endothelial en la diabetes. Diabetes Care. 2013;13(5):3-8.

9. Moreira ED Jr, Neves RC, Nunes ZO, de Almeida MC, Mendes AB, Fittipaldi JA, et al., Venezuelan Diabetes Investigators' Group. Glycemic control and its correlates in patients with diabetes in Venezuela: results from a nationwide survey. Diabetes Res Clin Pract. 2010;87(3):407-14.

10. De la Viga VM, Rodríguez MG, Quintana MG. La disfunción endotelial: una propuesta hacia el cambio [monografía en Internet]. Argentina Intermedicina; 2007 [consultado 12
Oct 2011]. Disponible en: http://www.intermedicina.com/ Publicaciones/Pub-08.htm

11. Velarde MS, Prado MM, Carrizo TR, Abregú AV, Sánchez SS. Evaluación de disfunción endotelial en pacientes con diabetes tipo 2. Acta Bioquím Clín Latinoam. 2007;41(4).

12. Morales VE, Di Sciascio G, Briguori C, Fuster V, Martínez M. Endotelio, aterotrombosis y estatinas. Atlas Ilustrado. Primera Edición México: Aguascalientes, Ags; 2010.

13. Alexánderson E, Calleja R, Martínez A, Lamothe PA, Ochoa JM, Meléndez G, et al. El impacto de la detección de disfunción endotelial en la aterosclerosis: estudio mediante tomografía por emisión de positrones. Arch Cardiol Méx. 2010;80(1):36-40.

14. Cruz HJ, Licea ME, Hernandez GP, Abraham ME, Yanes QM. Estrés oxidativo y diabetes mellitus. Rev Mex Patol Clin. 2011;58(1):4-15.

15. Díaz D. Hiperglicemia y estrés oxidativo en el paciente diabético. Rev Cub Invest Biomed. 2006;25(3):1-9.

16. Lapolla A, Reitano S, Seraglia R, Sartore G, Ragazzi E, Traldi P. Evaluation of advanced glycation end products and carbonyl compounds in patients with different conditions of oxidative stress. Mol Nutr Food Res. 2005;49(7):685-90.

17. Clapés S, Armas D, Marquetty A, Lemani M, Márquez I, Díaz D, et al. Disminución de la capacidad antioxidante en niños y adolescentes diabéticos. Rev Cub Invest Biomed. 2006;25(2):1-7.

18. Núñez RI, Socarrás EL, González Z, Chávez J, Cano C, Amell $A$, et al. Determinación de agentes antioxidantes séricos en diabéticos tipo 2. Med Interna (Caracas). 2001;17(4):1-10.

19. Bhatia S, Skukla R, Venkata Madhu S, Kaur Gambhir J, Madhava Prabhu K. Antioxidant status, lipid peroxidation and nitric oxide end products in patient of type 2 diabetes mellitus with nephropathy. Clin Biochem. 2003;36(7):557-62.

20. Lyons TJ. Glycation, carbonyl stress, EAGLEs, and the vascular complications of diabetes. Semin Vasc Med. 2002;2(2): 175-89.

21. Beisswenger PI, Drummond KS, Nelson RG, Howell SK, Szwergold BS, Mauer M. Susceptibility to diabetic nephropathy is related to dicarbonyl and oxidative stress. Diabetes. 2005;54(11):3274-81.

22. Du Y, Smith MA, Miller CM, Kern TS. Diabetes-induced nitrative stress in the retina, and correction by aminoguanidine. $J$ Neurochem. 2002;80(5):771-9.

23. Van Dam PS. Oxidative stress in diabetic neuropathy: pathophysiological mechanisms and treatment perspectives. Diabetes Metab Res Rev. 2002;18(3):176-264.

24. Feldman EL. Oxidative stress and diabetic neuropathy: a new understanding of an old problem. J Clin Invest. 2003;111(4):431-3.

25. Esteller A. Biología de la pared vascular y síndrome metabólico. Nutr Hosp. 2005; XX(1):5-17.

26. Johansen JS, Harris AK, Rychly DJ, Ergul A. Oxidative stress and the use of antioxidants in diabetes: Linking basic science to clinical practice. Cardiovasc Diabetol. 2005;4:5.

27. St Zatalia R, Sanusi H. The role of antioxidants in the pathophysiology, complications, and management of diabetes mellitus. Indones J Intern Med. 2013;45(2).

28. Ceriello A, Testa R. Antioxidant anti-inflammatory treatment in type 2 diabetes. Diab Care. 2009;32(2):S232-6.

29. Szabo C. Role of nitrosative stress in the pathogenesis of diabetic vascular dysfunction. Br J Pharmacol. 2009;156:713-27.

30. Rosiak M, Postuła M, Kapłon-Cieślicka A, Trzepla E, Filipiak KJ, Członkowski A, et al. The effect of doubling the dose of acetylsalicylic acid (ASA) on platelet function parameters in patients with type 2 diabetes and platelet hyperreactivity during treatment with $75 \mathrm{mg}$ of ASA: a subanalysis of the AVOCADO study. Kardiol Pol. 2013;71:552-7.

31. Wright RJ, Frier BM. Vascular disease and diabetes: is hypoglycaemia an aggravating factor? Diabetes Metab Res Rev. 2008;24:353-63. 
32. Rana OA, Byrne CD, Greaves K. Intensive glucose control and hypoglycaemia: a new cardiovascular risk factor? Heart. 2014;100:21-7.

33. Gogitidze Joy N, Hedrington MS, Briscoe VJ, Tate DB, Ertl AC, Davis SN. Effects of acute hypoglycemia on inflammatory and pro-atherothrombotic biomarkers in individuals with type 1 diabetes and healthy individuals. Diabetes Care. 2010;33: 1529-35.

34. Wright RJ, Macleod KM, Perros P, Johnston N, Webb DJ, Frier BM. Plasma endothelin response to acute hypoglycaemia in adults with type 1 diabetes. Diabet Med. 2007;24:1039-42.

35. Snell-Bergeon JK, Wadwa RP. Hypoglycemia, diabetes, and cardiovascular disease. Diabetes Technology \& Therapeutics. 2012;14 Suppl. 1.

36. Wang J, Alexanian A, Ying R, Kizhakekuttu TJ, Dharmashankar K, Vasquez-Vivar J, et al. Acute exposure to low glucose rapidly induces endothelial dysfunction and mitochondrial oxidative stress: role for AMP kinase. Arterioscler Thromb Vasc Biol. 2012;32:712-20.
37. Lucidi P, Rossetti P, Porcellati F, Pampanelli S, Candeloro P, Andreoli AM, et al. Mechanisms of insulin resistance after insulin-induced hypoglycemia in humans: the role of lipolysis. Diabetes. 2010;59:1349-57.

38. Nauck MA, Heimessat MM, Behle K, Holst JJ, Nauck MS, Ritzel $\mathrm{R}$, et al. Effects of glucagon-like peptide 1 on counterregulatory hormone responses, cognitive functions, and insulin secretion during hyperinsulinemic stepped hypoglycemic clamp experiments in healthy volunteers. J Clin Endocrin Metab. 2014;87(3):1239-46.

39. Ceriello A, Novials A, Ortega E, La Sala L, Pujadas G, Testa R, et al. Evidence that hyperglycemia after recovery from hypoglycemia worsens endothelial function and increases oxidative stress and inflammation in healthy control subjects and subjects with type 1 diabetes. Diabetes. 2012;61:2993-7.

40. Ceriello A, Novials A. Vitamin C further improves the protective effect of GLP-1 on the ischemia-reperfusion-like effect induced by hyperglycemia post-hypoglycemia in type 1 diabetes. Cardiovasc Diabetol. 2013. 